\title{
Single-mode Bragg Gratings in Tapered Few-Modes and Multimode Fibers
}

\author{
L. A. Herrera-Piad, ${ }^{1,}$ M. Delgado-Pinar, ${ }^{2}$ J. L. Cruz, ${ }^{2}$ A. Carrascosa, ${ }^{2}$ A. \\ DÍEZ, ${ }^{2}$ R. ROJAS-LAgunA, ${ }^{1}$ M. V. ANDRÉs ${ }^{2}$ \\ ${ }^{1}$ Department of Electronics, Universidad de Guanajuato, Campus Irapuato-Salamanca, Carretera Salamanca-Valle de Santiago km 3.5, \\ Salamanca, Gto. 36885, Mexico. \\ ${ }^{2}$ Department of Applied Physics, University of Valencia, Dr. Moliner 50, Burjassot 46100, Spain. \\ *Corresponding author: la.herrerapiad@ugto.mx
}

Received XX Month XXXX; revised XX Month, XXXX; accepted XX Month XXXX; posted XX Month XXXX (Doc. ID XXXXX); published XX Month XXXX

\begin{abstract}
We propose the implementation of fiber Bragg gratings in tapered few-modes, and multimode fibers to accomplish single-mode operation by reducing the core diameter while preserving the core-cladding structure. Gratings present a single reflection band and the device show low insertion losses after the taper fabrication and the fiber Bragg gratings inscription. The excitation of high order odd-modes in the core of the fiber has been identified as the main loss mechanism; it can be prevented by means of symmetric illumination of the fibers. We also demonstrate the excitation of high order cladding modes (cladding-air modes) along the taper transitions; these modes can be removed without a significant increment of the insertion loss. (C) 2018 Optical Society of America
\end{abstract}

http://dx.doi.org/10.1364/OL.99.099999

Telecommunications, laser and sensor technologies have been recently related with few-modes (FMF), Large Modal Area (LMA) and multimode fibers (MMF) [1-3]. More specifically, photonic lanterns, pump combiners and MMF have been newly proposed for high power lasers and communications $[4,5]$. Additionally, fiber Bragg gratings allow obtaining better efficiencies and stabilizing the emission spectrum of these laser devices [4-6]; some groups have demonstrated continuous wave lasers operating with multiple Bragg reflections [7-10], pursuing a narrower line width and increasing the number of laser lines. Bragg gratings in MMF have been proposed for spectral control of large diameter lasers, which can be difficult to couple with singlemode fibers (SMF) $[11,12]$. Furthermore, gratings in FMF and MMF are used for sensing by using high-order modes [13-15].

Tapered optical fibers are frequently used in lasers, sensing, and signal processing [16-22]. The fundamental modes of multimode and single-mode fibers can be efficiently matched by tapering the MMF $[23,24]$. These mode converters have been used to couple diode lasers to SMFs [24] and they could improve the performance of devices for dispersion management [25]. Gratings in tapered single-mode microfibers have been demonstrated [26], but the microfiber is in general fragile due to its reduced diameter, and their optical modes present large evanescent tails which are sensible to changes in the external medium; thus, they can present spurious noise. In addition, these gratings are more difficult to fabricate because of the fiber size and the lack of photosensitivity. Thus, applications are basically restricted to sensors.

There is a necessity of managing individual modes to improve the performance of some systems, and also to eliminate high order core modes to achieve single-mode operation in power fiber lasers and sensors. However, gratings in multimode or LMA fibers present several reflection bands due to the coupling between different propagating modes. Thus, unwanted reflections need to be removed to achieve single wavelength laser emission. In this work, we demonstrate single band reflectors made on tapered 4modes and tapered multimode fibers, with potential applications in fiber lasers. The fiber taper is designed to achieve low insertion loss, good guidance since it preserves the core-cladding structure, and fiber diameters still easy to handle. A weak excitation of cladding modes in the transitions of the taper has been observed; this makes the device potentially useful for chemical sensing.

Tapers were fabricated by the fuse-and-pull technique from commercial fibers, the fibers had low Germanium content and were hydrogenated at $30 \mathrm{Bar}$ and $22{ }^{\circ} \mathrm{C}$ for 15 days to increase their photosensitivity. Finally, gratings were photo-inscribed by a phase mask technique. A superluminescent diode was launched into a circulator to measure the gratings spectra as it is sketched in figure 1, polarized light was used in order to analyze possible birefringence in the tapers. The testing setup was made of singlemode fibers, with the exception of the device under test.

Two kinds of fibers were used in these experiments, a fourmodes step-index fiber (FMF) from OFS, and a multimode 50/125 graded index multimode fiber (GMMF) from Spectran Corporation; their estimated V-values at $1550 \mathrm{~nm}$ are 5 and 14, respectively. 

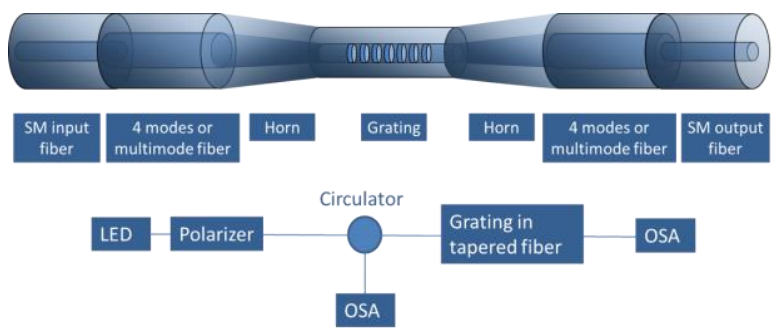

Fig. 1. Top: Diagram of a Bragg grating in a tapered fiber. Bottom: Testing setup.

It has been demonstrated in [24] and [25] that sufficiently long taper transitions can match the fundamental mode of a multimode fiber to the fundamental mode of a single mode fiber. In our work, tapers were fabricated with an exponential profile for the transitions; as it is shown in Fig. 2, which depicts the profile of several tapers of different diameters, fabricated from the GMMF. The uniform section of the taper (waist) had $40 \mathrm{~mm}$ in length to accommodate the gratings. The maximum length of the transitions was $50 \mathrm{~mm}$, for the case of the taper of $15 \mu \mathrm{m}$ in diameter. The profile of the tapers was measured with a laser gauge down to 25 $\mu \mathrm{m}$ diameters; smaller diameters were checked in an SEM microscope. The inset shows that single mode operation is expected when fiber diameters are below 55 (FMF), and $25 \mu \mathrm{m}$ (GMMF).

The insertion loss was measured in real time as the tapers were fabricated. Figure 3 shows the attenuation as a function of time (this is, as a function of the waist diameter) for a FMF and for a GMMF whose diameters were reduced to 50 and $15 \mu \mathrm{m}$, respectively. Both tapers become single mode as it will be demonstrated by means of the grating characterization. Each transition was made long enough to prevent sharp cut-off of high order modes and consequently diminish the insertion loss [24]. Measurements were made by exciting and collecting the light into the fibers with a single mode fiber. The power loss in $\mathrm{C}+\mathrm{L}$ bands is around $0.5 \mathrm{~dB}$ for the FMF and less than $1 \mathrm{~dB}$ for the GMMF.

The taper is excited by a SMF, the junction to the MMF generates symmetrical high-order core-modes, which in turn generate multiple spherical modes at the input of the taper transition [27], the spherical modes transfer energy to the fundamental mode in the transition as the diameter is reduced, and, finally, the output of the transition excites the single-mode waist. Therefore the taper transition works as an efficient impedance adapter of symmetric modes.

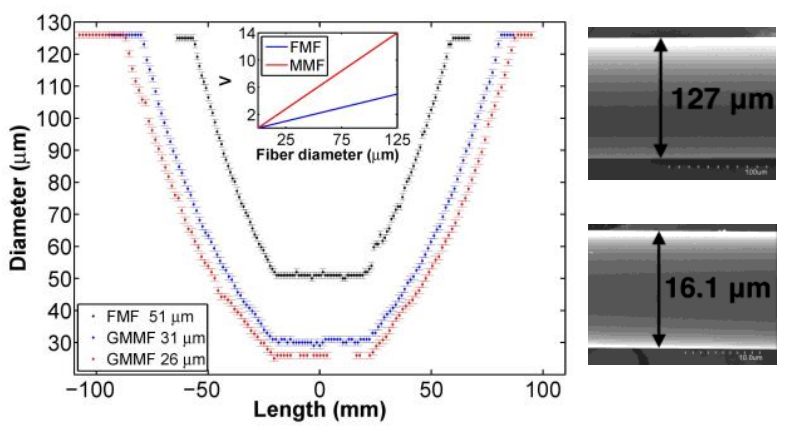

Fig. 2. Left, profiles of tapers of different diameters down to $25 \mu \mathrm{m}$. Inset, $\mathrm{V}$ value calculated as a function of the diameter. Right, SEM microscope pictures of a pristine fiber with $125 \mu \mathrm{m}$ of nominal diameter, and a taper waist of $15 \mu$ m of target diameter (127 and 16.1 actual values).

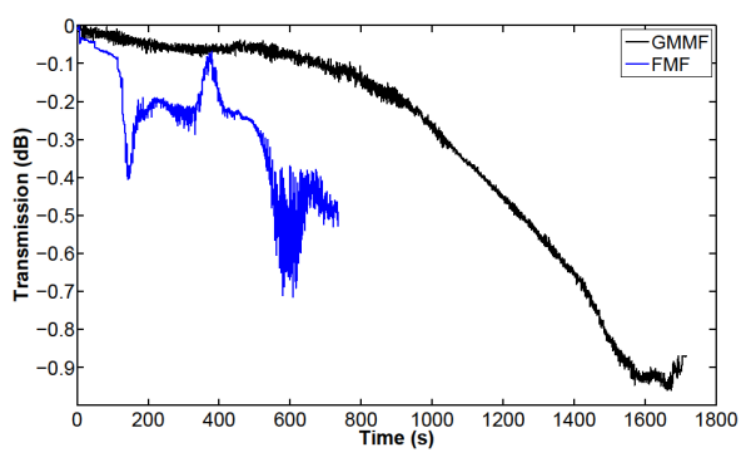

Fig. 3. Insertion loss of GMMF and FMF fiber tapers during fabrication.

The adapter is unable to match asymmetric core modes because the waist only guides the $\mathrm{LP}_{01}$ mode. To verify this, the GMMF was excited introducing a lateral offset between the GMMF and the SMF so that asymmetric modes are excited (asymmetric excitation). It can be seen in Fig. 4 that transmission falls below $35 \mathrm{~dB}$ for a $25 \mu \mathrm{m}$ lateral offset between both fiber cores. Transmission of Fig. 4 was measured directly in the GMMF (without output SMF).
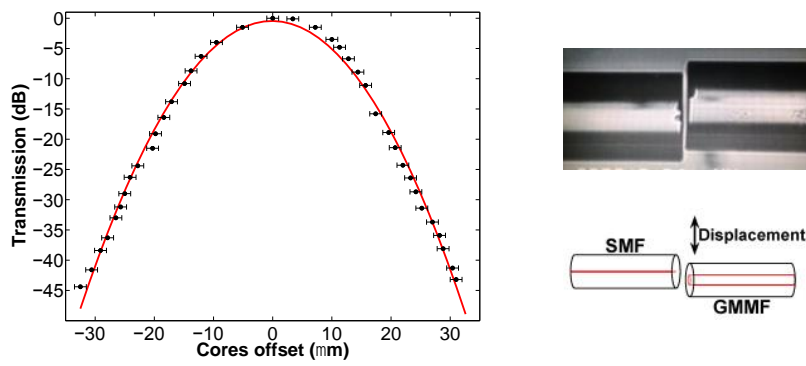

Fig. 4. Left: Transmission loss of the MMF taper when the core of the feeding SMF was misaligned with respect to the core of the GMM fiber (dots: experiment, line: parabolic fit). Right: Picture and diagram of the cores offset.

To proceed with the characterization of the gratings in MMFs, first we wrote a grating in a pristine FMF to find out the wavelength range and the relative strength of the different resonances correspondent to the coupling between the optical modes. Since the FMF holds four modes, a Bragg grating in the fiber should show ten resonances. The resonant wavelengths are given by the grating pitch $\Lambda$ and the effective indices $n_{\text {effi }}$ according to the coupling condition:

$$
\lambda_{i j}=\Lambda\left(n_{e f f \mathrm{i}}+n_{e f f \mathrm{j}}\right) \quad i, j \in\{1,2,3,4\}
$$

We used a phase mask with a period of $1076.25 \mathrm{~nm}$ and $2.5 \mathrm{~cm}$ of length for the gratings inscription. Asymmetric excitation and blazed gratings are required to observe the full set of resonances. Figure 5 (top) shows the spectrum of the tilted grating (tilt angle $2^{0}$ ), and the expected resonant wavelengths calculated from the eigenvalue-equation of the fiber [27]. Some resonances overlap: the coupling of the $\mathrm{LP}_{02}$ mode is degenerated with the crossed coupling between the $\mathrm{LP}_{02}$ and the $\mathrm{LP}_{21}$ modes; the same happens with the $\mathrm{LP}_{11}$ coupling and the crossed coupling $\mathrm{LP}_{01}-\mathrm{LP}_{21}$. The 
observation of the corresponding resonances depends on the illumination of the fiber, as well as on the symmetry of the gratings.
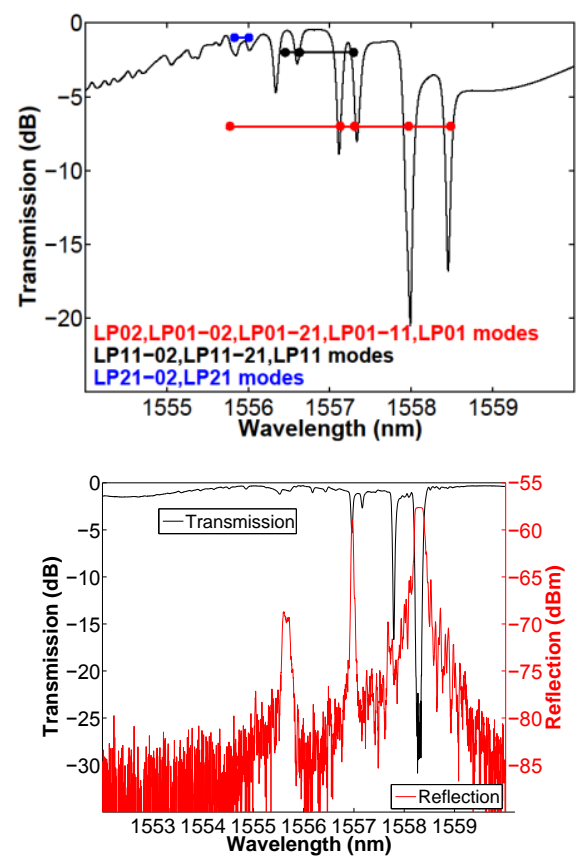

Fig. 5. Grating spectrum in pristine FMF. Top: tilted grating measured with asymmetric illumination, the dots show the calculated resonant wavelengths of LP modes coupling. Bottom: Standard grating with symmetric illumination.

The transmission level has an oscillation of $4 \mathrm{~dB}$ that is caused by the interference between modes, since light is injected with an offset between the fiber cores. When the grating is written without tilt and the illumination is symmetrical, there still appear some high order resonances that can hardly be distinguished from couplings to cladding modes, as it can be seen in figure 4 (bottom); furthermore, the oscillation in the transmitted power level is reduced to less than $2 \mathrm{~dB}$. The reflection spectrum shows the resonances corresponding to symmetric modes $\left(\mathrm{LP}_{01}-\mathrm{LP}_{01}, \mathrm{LP}_{01}\right.$ $\mathrm{LP}_{02}$ and $\mathrm{LP}_{02}-\mathrm{LP}_{02}$ ). The transmission spectrum still presents the $\mathrm{LP}_{01}-\mathrm{LP}_{11}$ resonance because of the strength of the grating and its residual asymmetry. Even modes have weaker but not null resonances, due to the residual asymmetries in the gratings; some resonances can hardly be observed in reflection because the splice between the feeding SMF and the FMF acts as a modal filter.

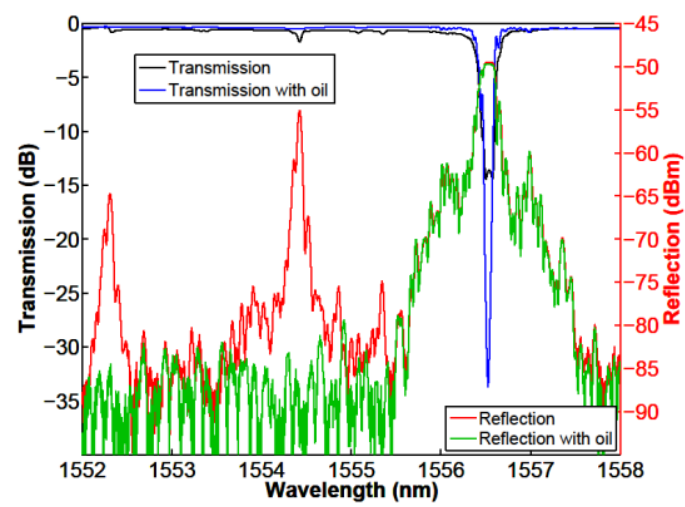

Fig. 6. Spectra of a Bragg grating in a tapered FMF. Transmission and reflection have been measured with the fiber surrounded by air (black and red lines) and by an index matching fluid (oil with $n=1.456$, blue and green lines).

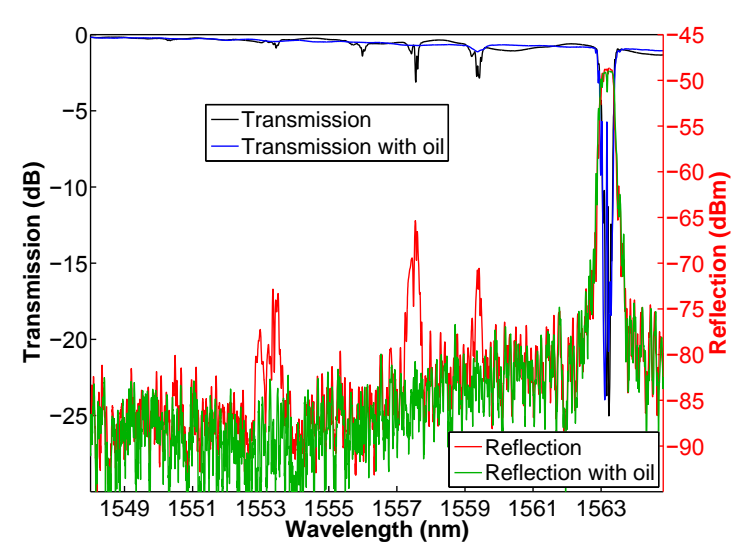

Fig. 7. Spectra of a Bragg grating in a tapered GMMF. Transmission and reflection have been measured with the fiber surrounded by air (black and red lines) and by an index matching fluid ( $\mathrm{n}=1.456$, blue and green lines).

The FMF was first tapered to achieve single mode operation; after that, a standard Bragg grating was written in the taper waist. The tapered fiber waist had $50 \mu \mathrm{m}$ in diameter-and $40 \mathrm{~mm}$ in length (V-value about 2, see Fig. 2). The measured spectra with symmetric illumination are presented in Fig. 6.

Besides the fundamental resonance at $1556.5 \mathrm{~nm}$, the grating as written produces other small resonances that can be clearly observed in the reflection spectrum. These reflections, centered at 1552 and $1554 \mathrm{~nm}$, do not correspond to high order core modes because they can be removed by covering the fiber with an index matching oil to match the refractive index of the external medium to that of the fiber cladding; thus, these resonances correspond to couplings involving cladding modes propagating in the waist of the taper, since they are sensitive to the presence of the oil.

What is more interesting is that these resonances are not due to the standard coupling from the fundamental core mode to cladding modes that are usually observed in FBGs written in SMF. This mechanism of mode excitation cannot be observed in reflection (cladding modes are suppressed by the FMF coating [28]). Therefore, the origin of these cladding modes reflected by the core is outside of the grating. We believe that these cladding modes are generated in the input taper transition, that couples energy from the core modes of the fiber to cladding modes. When these cladding modes interact with the grating, their energy is transferred to contrapropagating core modes that can be observed in reflection at the output of the FMF.

The removal of the cladding modes by means of the matching index oil does not affect significantly the transmitted power level. Notice that the depth of the fundamental resonance is fully resolved (rejection higher than $30 \mathrm{~dB}$ ) when the fiber is index matched (see Fig. 6, transmission spectrum, blue line). On the contrary, when cladding modes are present (see Fig. 6, transmission, black line), the notch is about $-13 \mathrm{~dB}$ deep. Therefore, the energy carried by cladding modes must be less than $5 \%$; this number is in agreement with the interferometric variation of the transmission level out of resonance (less than $1 \mathrm{~dB}$ ).

To validate the good performance of our technique in fibers with a large number of modes, we repeated the process using the GMMF (estimated V value: 14). The same procedure for the taper fabrication was used. In this case, the fiber diameter is reduced further and, consequently, the taper transition is slightly longer (50 
$\mathrm{mm}$ as a maximum). Single mode operation was observed for diameters below $25 \mu \mathrm{m}$. Figure 7 presents the spectra of a grating inscribed in the taper waist (taper waist: $40 \mathrm{~mm}$ long and $20 \mu \mathrm{m}$ in diameter). Transmission and reflection spectra have been measured with and without external index matching.

The spectra show the reflection of the fundamental core mode (centered at $1563 \mathrm{~nm}$ ) as well as other resonances at shorter wavelengths. Like in the FMF case, the sidebands can be removed by matching the external refractive index, which is an indication of single-mode core operation. Again, cladding light is excited by the input taper transition, before the grating. We have not observed a significant variation of the transmission level when the fiber is covered with matching oil, therefore the energy level in the cladding modes is lower in GMMF tapers than in FMF tapers. We have also observed in many tapers made in both fibers, that the power level in the cladding modes is more repetitive in GMMF than in FMF. This happens because the fusion splice between the SMF feeding fiber and FMF is more sensitive to misalignment that the splice to the GMMF (in terms of high order modes generation).

Finally, we report in Fig. 8 the spectra of gratings made in GMMF tapers of different diameters between 15 and $20 \mu \mathrm{m}$. All gratings have the same length and have been written with the same UV exposure conditions. The reduction of the $\mathrm{V}$-value as the fiber diameter is reduced results in a smaller overlap integral and, therefore, in less reflective gratings. The spectra always present some chirp, no polarization dependence has been observed; the chirp is due to geometrical imperfections or to index fluctuations in the tapers, we believe it can be improved pulling the fibers with a faster tapering rig and at controlled temperature.

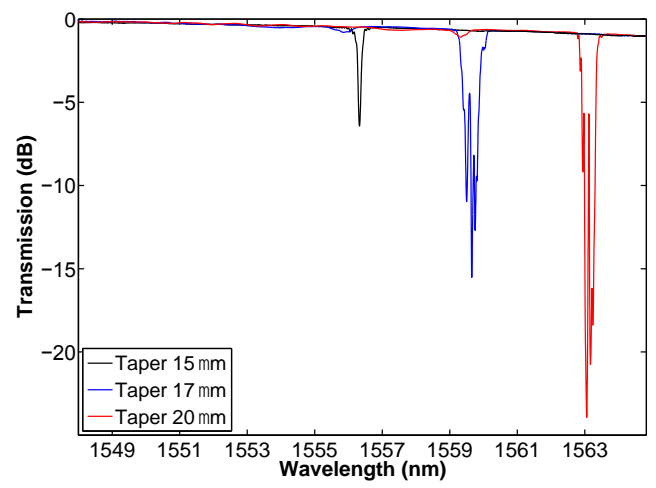

Fig. 8. Transmission spectra of the Bragg gratings in the tapered MMF with three different diameters. Fibers are surrounded by index matching oil.

Summarizing, in this work efficient single mode Bragg reflectors have been demonstrated in tapered few-mode and multimode fibers. The taper waist preserves the core-cladding structure, hence it ensures a reasonable mechanical strength and immunity to the surrounding media for the core mode. We have identified the coupling of energy to cladding modes in the input transition of the taper. The gratings reflect these cladding modes, and produce transmission/reflection bands that are sensitive to the surrounding index, paving the way for sensor applications. Reflectivities of more than $99.7 \%$ have been achieved at the fundamental resonances. The insertion loss of the devices is kept below $1 \mathrm{~dB}$, this figure could be even smaller if the device were illuminated with a pure $\mathrm{LP}_{01}$ mode in a laser cavity of a multimode fiber.
Funding. CONACYT student scholarship 546217/300665; Project SEP-PRODEP UGTO-PTC541. Ministerio de Economía y Competitividad de España and FEDER (TEC2016-76664-C2-1-R).

\section{References}

1. Q. Sui, H. Zhang, J. D. Downie, W. A. Wood, J. Hurley, S. Mishra, A. P. T. Lau, C. Lu, H.-Y. Tam, and P. K. A. Wai, Opt. Express 23, 3156 (2015).

2. F. Beier, C. Hupel, J. Nold, S. Kuhn, S. Hein, J. Ihring, B. Sattler, N. Haarlammert, T. Schreiber, R. Eberhardt, and A. Tünnermann, Opt. Express 24, 6011 (2016).

3. J. Montoya, C. Hwang, D. Martz, C. Aleshire, T. Y. Fan, and D. J. Ripin, Opt. Express 25, 27543 (2017).

4. L. V. Kotov, S. S. Aleshkina, M. M. Khudyakov, M. M. Bubnov, O. I. Medvedkov, D. S. Lipatov, A. N. Guryanov, and M. M. Likhachev, J. Light. Technol. 35, 4540 (2017).

5. S. G. Leon-Saval, T. A. Birks, J. Bland-Hawthorn, and M. Englund, Opt. Lett. 30, 2545 (2005).

6. A. Brown, P. Leisher, G. Fanning, S. Lerner, Z. Chen, L. Bao, M. Grimshaw, M. DeVito, K. Price, K. Kennedy, S. Lin, M. Reynolds, S. Karlsen, J. Small, R. Martinsen, and J. Haden, in Proceedings of SPIE (2011), p. 803900.

7. Y. Liu, X. Feng, S. Yuan, G. Kai, and X. Dong, Opt. Express 12, 2056 (2004).

8. X. Liu, X. Zhou, X. Tang, J. Ng, J. Hao, T. Y. Chai, E. Leong, and C. Lu, IEEE Photonics Technol. Lett. 17, 1626 (2005).

9. X. Feng, H. Tam, S. Member, and P. K. A. Wai, IEEE Photonics Technol. Lett. 18, 1088 (2006).

10. X. Liu, X. Yang, F. Lu, J. Ng, X. Zhou, and C. Lu, Opt. Express 13, 142 (2005).

11. T. Mizunami, T. V. Djambova, T. Niiho, and S. Gupta, J. Light. Technol. 18, 230 (2000).

12. Y. Sun, T. Szkopek, and P. W. E. Smith, Opt. Commun. 223, 91 (2003).

13. W. Jin, Y. Xu, Y. Jiang, Y. Wu, S. Yao, S. Xiao, Y. Qi, W. Ren, and S. Jian, Laser Phys. 28, (2018).

14. X. Sang, C. Yu, B. Yan, T. Mayteevarunyoo, K. Wang, and N. Lu, Microw. Opt. Technol. Lett. 48, 1739 (2006).

15. A. Sun and Z. Wu, IEEE Sens. J. 15, 3390 (2015).

16. H. Ahmad and A. A. Jasim, Opt. Fiber Technol. 36, 105 (2017).

17. K. Kieu and M. Mansuripur, Opt. Lett. 32, 2242 (2007).

18. J. A. Alvarez-Chavez, A. B. Grudinin, J. Nilsson, P. W. Turner, and W. A. Clarkson, Proc. CLEO 247 (1999).

19. N. H. Zainuddin, H. Y. Chee, M. Z. Ahmad, M. A. Mahdi, M. H. Abu Bakar, and M. H. Yaacob, J. Biophotonics e201700363, 12 (2018).

20. L. A. Herrera-Piad, D. Jauregui-Vazquez, Y. Lopez-Dieguez, J. M. Estudillo-Ayala, J. C. Hernandez-Garcia, J. M. Sierra-Hernandez, M. Bianchetti, and R. Rojas-Laguna, Laser Phys. 28, 7 (2018).

21. T. Osuch, Opt. Commun. 366, 194 (2016).

22. S.M. Popov, O.V. Butov, Y.K. Chamorovskiy, V.A. Isaev, A.O. Kolosovskiy, V.V. Voloshin, I.L. Vorob'ev, M.Yu. Vyatkin, P. Mégret, M. Odnoblyudov, D.A. Korobko, I.O. Zolotovskii, and A.A. Fotiadi, Results in Phys. 9, 625 (2018).

23. Y. Yang, J. Lee, K. Reichard, P. Ruffin, F. Liang, D. Ditto, and S. Yin, Opt. Commun. 249, 129 (2005).

24. A. E. Ash, M. W. Austin, and J. D. Love, Opt. Quantum Electron. 32, 197 (2000).

25. Z. Wu, J. Li, Y. Tian, D. Ge, J. Zhu, F. Ren, Q. Mo, J. Yu, Z. Li, Z. Chen, and, Y. He, Opt. Commun. 410, 112 (2018).

26. Y. Liu, C. Meng, A. Ping, Y. Xiao, H. Yu, and, L. Tong, Opt. Lett. 36, 3115 (2011).

27. C. A. Balanis, Advanced engeneering electromagnetics, John Wiley \& Sons (2012).

28. D. Sáez-Rodriguez, J. L. Cruz, A. Díez, and M. V. Andrés, Opt. Lett. 36, 1518 (2011). 
References with titles:

1. Q. Sui, H. Zhang, J. D. Downie, W. A. Wood, J. Hurley, S. Mishra, A. P. T. Lau, C. Lu, H.-Y. Tam, and P. K. A. Wai, "Long-haul quasi-singlemode transmissions using few-mode fiber in presence of multipath interference" Opt. Express 23, 3156 (2015).

2. F. Beier, C. Hupel, J. Nold, S. Kuhn, S. Hein, J. Ihring, B. Sattler, N. Haarlammert, T. Schreiber, R. Eberhardt, and A. Tünnermann, "Narrow linewidth, single mode $3 \mathrm{~kW}$ average power from a directly diode pumped ytterbium-doped low NA fiber amplifier" Opt. Express 24, 6011 (2016).

3. J. Montoya, C. Hwang, D. Martz, C. Aleshire, T. Y. Fan, and D. J. Ripin, "Photonic lantern kW-class fiber amplifier" Opt. Express 25, 27543 (2017).

4. L. V. Kotov, S. S. Aleshkina, M. M. Khudyakov, M. M. Bubnov, O. I. Medvedkov, D. S. Lipatov, A. N. Guryanov, and M. M. Likhachev, "High-brightness multimode fiber lasers for resonant pumping," J. Light. Technol. 35, 4540 (2017).

5. S. G. Leon-Saval, T. A. Birks, J. Bland-Hawthorn, and M. Englund, "Multimode fiber devices with single-mode performance," Opt. Lett. 30, 2545 (2005).

6. A. Brown, P. Leisher, G. Fanning, S. Lerner, Z. Chen, L. Bao, M. Grimshaw, M. DeVito, K. Price, K. Kennedy, S. Lin, M. Reynolds, S. Karlsen, J. Small, R. Martinsen, and J. Haden, "Progress in commercial wavelength-stabilized high-brightness diode sources suitable for pumping Yb-doped fiber lasers," in Proceedings of SPIE (2011), p. 803900.

7. Y. Liu, X. Feng, S. Yuan, G. Kai, and X. Dong, "Simultaneous fourwavelength lasing oscillations in an erbium-doped fiber laser with two high birefringence fiber Bragg gratings, "Opt. Express 12, 2056 (2004).

8. X. Liu, X. Zhou, X. Tang, J. Ng, J. Hao, T. Y. Chai, E. Leong, and C. Lu, "Switchable and tunable multiwavelength erbium-doped fiber laser with fiber Bragg gratings and photonic crystal fiber,"IEEE Photonics Technol. Lett. 17, 1626 (2005).

9. X. Feng, H. Tam, S. Member, and P. K. A. Wai, "Switchable Multiwavelength Erbium-Doped Fiber Laser With a Multimode Fiber Bragg Grating and Photonic Crystal Fiber," IEEE Photonics Technol. Lett. 18, 1088 (2006).

10. X. Liu, X. Yang, F. Lu, J. Ng, X. Zhou, and C. Lu, "Stable and uniform dual-wavelength erbium-doped fiber laser based on fiber Bragg gratings and photonic crystal fiber,"Opt. Express 13, 142 (2005).

11. T. Mizunami, T. V. Djambova, T. Niiho, and S. Gupta, "Bragg gratings in multimode and few-mode optical fibers,"J. Light. Technol. 18, 230 (2000).

12. Y. Sun, T. Szkopek, and P. W. E. Smith, "Demonstration of narrowband high-reflectivity Bragg gratings in a novel multimode fiber, "Opt. Commun. 223, 91 (2003).

13. W. Jin, Y. Xu, Y. Jiang, Y. Wu, S. Yao, S. Xiao, Y. Qi, W. Ren, and S. Jian, "Strain and temperature characteristics of the LP11 mode based on a few-mode fiber Bragg grating and core-offset splicing, "Laser Phys. 28, (2018).

14. X. Sang, C. Yu, B. Yan, T. Mayteevarunyoo, K. Wang, and N. Lu, "Experimental investigation on chemical sensor based on multimoe fiber Bragg grating" Microw. Opt. Technol. Lett. 48, 1739 (2006).

15. A. Sun and Z. Wu, "Multimode Interference in Single Mode Multimode FBG for Simultaneous Measurement of Strain and Bending,"IEEE Sens. J. 15, 3390 (2015).

16. H. Ahmad and A. A. Jasim, "Stable C-band fiber laser with switchable multi-wavelength output using coupled microfiber Mach-Zehnder interferometer,"Opt. Fiber Technol. 36, 105 (2017).

17. K. Kieu and M. Mansuripur, "Femtosecond laser pulse generation with a fiber taper embedded in carbon nanotube/polymer composite," Opt. Lett. 32, 2242 (2007).

18. J. A. Alvarez-Chavez, A. B. Grudinin, J. Nilsson, P. W. Turner, and W.
A. Clarkson, "Mode selection in high power cladding pumped fibre lasers with tapered section," Proc. CLEO 247 (1999).

19. N. H. Zainuddin, H. Y. Chee, M. Z. Ahmad, M. A. Mahdi, M. H. Abu Bakar, and M. H. Yaacob, "Sensitive Leptospira DNA detection using tapered optical fiber sensor," "J. Biophotonics e201700363, 12 (2018).

20. L. A. Herrera-Piad, D. Jauregui-Vazquez, Y. Lopez-Dieguez, J. M. Estudillo-Ayala, J. C. Hernandez-Garcia, J. M. Sierra-Hernandez, M. Bianchetti, and R. Rojas-Laguna, "Highly stable multi-wavelength erbium-doped fiber linear laser based on modal interference," Laser Phys. 28, 7 (2018).

21. T. Osuch, "Tapered and linearly chirped fiber Bragg gratings with codirectional and counter-directional resultant chirps," Opt. Commun. 366, 194 (2016).

22. S.M. Popov, O.V. Butov, Y.K. Chamorovskiy, V.A. Isaev, A.O. Kolosovskiy, V.V. Voloshin, I.L. Vorob'ev, M.Yu. Vyatkin, P. Mégret, M. Odnoblyudov, D.A. Korobko, I.O. Zolotovskii, A.A. Fotiadi, "Brillouin lasing in single-mode tapered optical fiber with inscribed fiber Bragg grating array," Results in Phys. 9, 625 (2018).

23. Y. Yang, J. Lee, K. Reichard, P. Ruffin, F. Liang, D. Ditto, and S. Yin, "Fabrication and implementation of a multi-to-single mode converter based on a tapered multimode fiber," Opt. Commun. 249, 129 (2005).

24. A. E. Ash, M. W. Austin, and, J. D. Love, "Efficient coupling of a laser to a waveguide using a taper designed by conformal mapping," Opt. Quantum Electron. 32, 197 (2000).

25. Z. Wu, J. Li, Y. Tian, D. Ge, J. Zhu, F. Ren, Q. Mo, J. Yu, Z. Li, Z. Chen, and, Y. He, "Fundamental-mode MMF transmission enabled by mode conversion,"Opt. Commun. 410, 112 (2018).

26. Y. Liu, C. Meng, A. Ping, Y. Xiao, H. Yu, and L. Tong, "Compact microfiber Bragg gratings with high-index contrast," Opt. Lett. 36, 3115 (2011).

27. C. A. Balanis, "Advanced engeneering electromagnetics," John Wiley \& Sons (2012).

28. D. Sáez-Rodriguez, J. L. Cruz, A. Díez, and, M. V. Andrés, "Coupling between counter-propagating cladding modes in fiber Bragg gratings," Opt. Lett. 36, 1518 (2011). 